\title{
Interferences in the Transformation of Reference Frames during a Posture Imitation Task
}

\author{
Eric L. Sauser and Aude G. Billard \\ Learning Algorithms and Systems Laboratory, LASA \\ Ecole Polytechnique Fédérale de Lausanne, EPFL, Switzerland \\ \{eric.sauser, aude.billard\}@epfl.ch
}

\begin{abstract}
We present a biologically-inspired neural model addressing the problem of transformations across frames of reference in a posture imitation task. Our modeling is based on the hypothesis that imitation is mediated by two concurrent transformations selectively sensitive to spatial and anatomical cues. In contrast to classical approaches, we also assume that separate instances of this pair of transformations are responsible for the control of each side of the body. We also devised an experimental paradigm which allowed us to model the interference patterns caused by the interaction between the anatomical on one hand, and the spatial imitative strategy on the other hand. The results from our simulation studies thus provide predictions of real behavioral responses.
\end{abstract}

\section{Introduction}

Although imitation has been extensively addressed in developmental psychology, it has become a current topic in neuroscience and experimental psychology $[1,3,4$, $8,9,11]$. The starting point of these investigations was the discovery, in monkey and human brain, of mirror neurons (MN), which are activated by both the execution and the observation of goal-directed actions [1,9,11]. In humans, the MN circuit, gets also activated during the presentation of meaningless gestures or body postures [9]. In this work, we focus on the process of transformation across frames of reference, required for imitation of arbitrary gestures. In psychology, anatomical and spatial types of imitation are usually considered distinct $[5,8$, 9]. On one hand, anatomical imitation considers the observed movements with respect to the observed person's body. On the other hand, spatial imitation considers only the spatial location of the limbs with respect to the observer, regardless of the orientation of the demonstrator. When the imitator and the demonstrator are facing each other, this form of imitation is usually denoted as specular or mirror $[4,5,9]$.

We hypothesize that the computations associated with these two forms of imitation are simultaneously computed in the brain. Given the task constraints, a competitive process then selects the correct response $[4,5,12]$. Such a competition usually produces measurable interferences on reaction times $[4,5,8]$. In addition to the previous hypothesis, we suggest that anatomical imitation should not be considered too strictly. We propose here that an anatomical mapping between contralateral limbs, which mirrors the relationship between the limb joint, also exists. Therefore, our model assumes that distinct pairs of spatial and anatomical 
transformations are responsible of the control of each side of the body. As a consequence, when an arm posture is presented with either the left or the right arm for example, an imitative response is computed in parallel for both arms of the imitator. Here, we apply a biologically-inspired modeling approach, known as the Dynamic Field Theory [7,12], to the problem of conflicting transformations across frames of reference. We will first present an experimental paradigm which will help determine the interferences between different imitative strategies during a task requiring the imitation of meaningless body postures. Then, we will briefly describe a neural model, capable of computing both anatomical and spatial imitative transformations. Finally, we will discuss the particular interference patterns predicted by our model and their implications for future research.

\section{Model}

\subsection{Experimental Scenario and Setup}

We consider the imitation of body postures where the orientation of the right upper arm is varied. The visual perspective of the demonstrator's body can also vary from side to front view. The task instructions require either a spatial or an anatomical imitative response with either the right arm (the corresponding one) or the left arm (the opposite one). The stimulus variables, shown in Figure 1, are: $\varphi^{D}$, the demonstrator arm elevation, $\theta^{D}$, its orientation relative to the body in the horizontal plane, and $\phi^{D}$, the orientation of the body with respect to the observer. The response variables are: $\varphi_{L}^{I}$ and $\varphi_{R}^{I}$, the elevation of the left and right arm of the imitator, and $\theta_{L}^{I}$ and $\theta_{R}^{I}$, their orientations on the horizontal plane. The desired responses are:

$$
\begin{aligned}
& \theta_{L}^{I, \mathrm{~A}}=\theta_{R}^{I, \mathrm{~A}}=\theta^{D} \\
& \theta_{L}^{I, \mathrm{~S}}=-\theta_{R}^{I, \mathrm{~S}}=\left\{\begin{array}{rr}
-180-\left(\theta^{D}+\phi^{D}\right) & \theta^{D}+\phi^{D}<-90 \\
\theta^{D}+\phi^{D} & \left|\theta^{D}+\phi^{D}\right| \leq 90 \\
180-\left(\theta^{D}+\phi^{D}\right) & \theta^{D}+\phi^{D}>90
\end{array}\right.
\end{aligned}
$$

where the additional index, $\mathrm{A}$ or $\mathrm{S}$, denotes the anatomical or the spatial imitative strategy, respectively. An illustration of these transformations are shown in Figure 2. Let us then define the discrepancy $D$ between the response of both strategies, which is is given by the difference between the response of the instructed strategy and that of the other. Spatial and anatomical transformations are said to be perfectly congruent when the discrepancy $D=0$. Note that ideal congruency conditions are not equivalent for both arms.

An experimental trial consists first of the presentation of a starting posture which the model is requested to imitate according to the task instructions. Then, 
the arm posture is abruptly changed, and the subject has to keep imitating as fast as possible. During a single trial, only the arm posture is modified, whereas the body orientation is left unchanged. Experiment 1 investigates the interferences produced by both imitative strategies when their initial responses are congruent and the amplitude of the change of arm posture is kept constant across the trials. The pair of initial and target postures consists of the arm raising from a neutral down position $\left(\varphi^{D}=0^{\circ}\right)$, where the responses of both transformations are always congruent, to a position on the horizontal plane $\left(\varphi^{D}=90^{\circ}\right)$. The arm elevation is thus the only degree of freedom which changes during a trial. Complementarily, Experiment 2 investigates the influence of a horizontal postural change, which amount is denoted by $\Delta \theta^{D}$. Indeed, in such conditions, depending on the stimuli, the discrepancy between the responses of the transformations may vary.

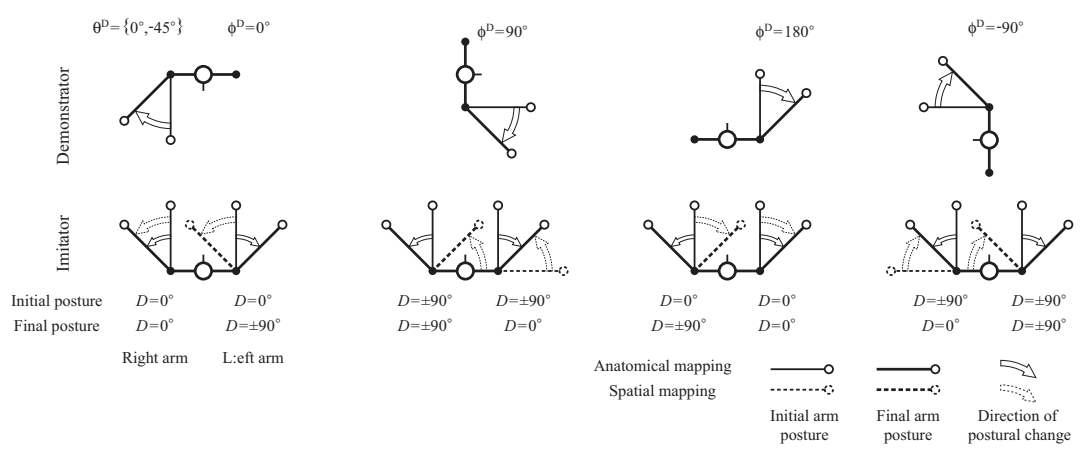

Fig. 2. Examples of anatomical and spatial imitative strategies in various conditions. The discrepancy between the response of the transformations are given for each arm.

\subsection{Neural fields}

This section briefly describes our model, which is composed of networks known as neural fields [7,12]. Formally, a neural field is composed of a continuous set of neurons, where each of them fires maximally for a specific value $\boldsymbol{r}$ uniformly distributed in the parameter space $\Gamma$. Since the modeled variables consist of arm and body orientations, we consider the parameter space as the ensemble of directions in the three dimensional space, i.e, $\Gamma=\left\{\boldsymbol{r} \in \mathbb{R}^{3} \mid\|\boldsymbol{r}\|=1\right\}$. Each neuron of the network is fully connected by means of recurrent synaptic weights $W^{R}$, exhibiting symmetry, rotational invariance and center-surround characteristics. The neural field dynamics follows

$$
\tau \dot{u}(\boldsymbol{r}, t)=-u(\boldsymbol{r}, t)+x(\boldsymbol{r}, t)+h(t)+\oint_{\Gamma} W^{R}\left(\boldsymbol{r}^{\prime}, \boldsymbol{r}\right) f\left(u\left(\boldsymbol{r}^{\prime}, t\right)\right) \mathrm{d} \boldsymbol{r}^{\prime}
$$

where $u(\boldsymbol{r}, t) \in \mathbb{R}$ is the membrane potential of the neuron with time constant $\tau \in$ $\mathbb{R}$ and the preferred direction $\boldsymbol{r}$ at time $t . f(y)=\max (0, y), x(\boldsymbol{r}, t)$ corresponds to the external input and $h(t)$ to a global modulatory input. The weight linking two neurons, with preferred directions $\boldsymbol{r}^{\prime}$ and $\boldsymbol{r}$, is given by a periodical Gaussian-like profile defined as

$$
W^{R}\left(\boldsymbol{r}^{\prime}, \boldsymbol{r}\right)=\alpha^{R}\left(g\left(\boldsymbol{r}^{\prime}, \boldsymbol{r}\right)-1\right) \quad \text { where } \quad g\left(\boldsymbol{r}^{\prime}, \boldsymbol{r}\right)=\frac{1}{\kappa} \exp \left(\frac{\left(\boldsymbol{M} \boldsymbol{r}^{\prime}\right)^{T} \boldsymbol{r}-1}{2 \sigma^{2}}\right)(3)
$$


$\alpha^{R}>0$ and $\sigma>0$ are, respectively, the amplitude and variance of the weight profile. $\boldsymbol{M}$ refers to a transformation or mapping matrix. In this case $\boldsymbol{M}=\boldsymbol{I}$, i.e., the identity matrix, but different mappings will be described later in the text. $\kappa=$ $1-e^{-\frac{1}{\sigma^{2}}}$ is a normalization factor ensuring that $g\left(\boldsymbol{r}^{\prime}, \boldsymbol{r}\right) \in$ $[0,1]$. This type of neural dynamics is known to form an attractor bump on the surface of the neural field (see Fig. 3),

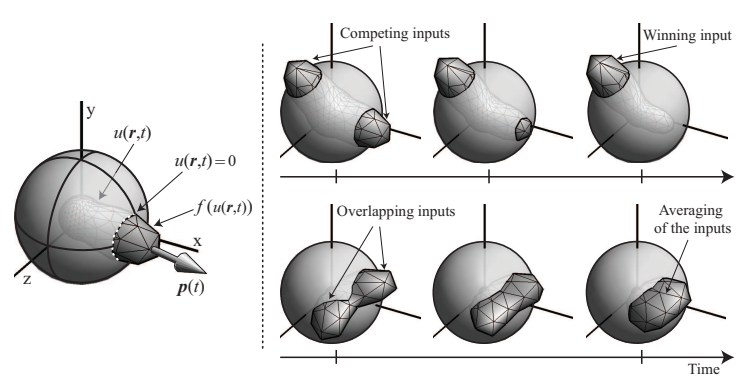

Fig. 3. (Left) Graphical representation of a neural field activity. (Right) Evolution of the neural activity during a selection process involving two competing inputs (top), and between two partially overlapping inputs (bottom).

through which this class of networks is suggested to convey information. As a read-out mechanism, we consider the population vector $\hat{\boldsymbol{p}}(t) \in \Gamma$. It consists of a weighted summation of the firing rate of each neuron with its preferred direction and is given by

$$
\hat{\boldsymbol{p}}(t)=\boldsymbol{p}(t) /\|\boldsymbol{p}(t)\| \quad \text { where } \quad \boldsymbol{p}(t)=\oint_{\Gamma} f(u(\boldsymbol{r}, t)) \boldsymbol{r} \mathrm{d} \boldsymbol{r}
$$

Moreover, we define $E(t)=\|\boldsymbol{p}(t)\|$ as the energy of the network response. Since further in the text we consider several neural fields within a large network, we will denote with an index $i$ the network variables corresponding to those of a neural field $i$. The external input $x^{i}(\boldsymbol{r}, t)$ can be composed of a direct sensory input and of synaptic projections from one or several different neural fields. A sensory input is constrained to represent a variable value $s(t) \in \Gamma$, and projection from a population $j$ to a population $i$ is done through synaptic weights $W^{j i}$. The external input of a neural field $i$ is then written as

$$
\begin{array}{r}
x^{i}(\boldsymbol{r}, t)=\beta^{i}\left(g\left(\boldsymbol{r}, \boldsymbol{s}^{i}(t)\right)-\eta\right)+\sum_{j} \oint_{\Gamma} W^{j i}\left(\boldsymbol{r}^{\prime}, \boldsymbol{r}\right) f\left(u^{j}\left(\boldsymbol{r}^{\prime}, t\right)\right) \mathrm{d} \boldsymbol{r}^{\prime} \quad \text { where } \\
W^{j i}\left(\boldsymbol{r}^{\prime}, \boldsymbol{r}\right)=\alpha^{j i}\left(g\left(\boldsymbol{r}^{\prime}, \boldsymbol{r}\right)-\eta^{j i}\right)
\end{array}
$$

where $\beta^{i}$ is the strength of the representation of the sensory variable $\boldsymbol{s}^{i}(t), \eta^{j i}$ is a normalization term, and $\alpha^{j i}>0$ is the amplitude of the weights. Similarly, the modulatory input $h^{i}(t)$ can consist of a constant input or of synaptic projections from other neural populations. In the latter case, we have

$$
h^{i}(t)=\sum_{j} \oint_{\Gamma} W^{j i}\left(\boldsymbol{r}^{\prime}, \boldsymbol{r}^{i}\right) f\left(u^{j}\left(\boldsymbol{r}^{\prime}, t\right)\right) \mathrm{d} \boldsymbol{r}^{\prime}
$$

where $\boldsymbol{r}^{i} \in \Gamma$ is constant and $W^{j i}$ corresponds to that defined in Equ. (5).

\subsection{Network Architecture}

The model architecture, depicted in Figure 4, consists of two networks, one for each arm. Within a single network, two main streams process separately the 


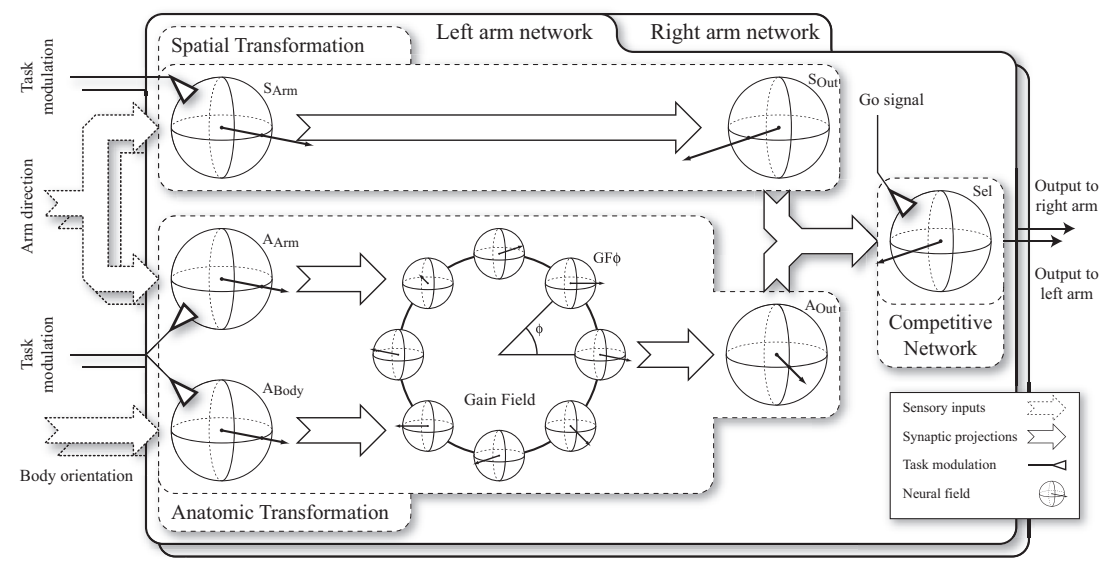

Fig. 4. Architecture of the model: Within each network corresponding to a given arm, two streams compute separately the anatomical and the spatial transformations.

spatial and the anatomical transformation. Their outputs are projected to a competitive network to select the appropriate response. Since the task instructions specify which arm should be used, the model does not perform the selection of the effector.

As external inputs, the two streams receive visual input in the form of the arm and body orientation vectors $s^{\text {Arm }}$ and $s^{\text {Body }} \in \Gamma$, relative to the reference frame of the observer (shown in Fig. 1):

$$
\begin{aligned}
\boldsymbol{s}^{\mathrm{Arm}} & =\left(\sin \left(\varphi^{D}\right) \sin \left(\theta^{D}+\phi^{D}\right), \cos \left(\varphi^{D}\right),-\sin \left(\varphi^{D}\right) \cos \left(\theta^{D}+\phi^{D}\right)\right)^{T} \\
\boldsymbol{s}^{\text {Body }} & =\left(\sin \left(\phi^{D}\right) \quad, 0 \quad,-\cos \left(\phi^{D}\right)\right.
\end{aligned}
$$

These inputs are fed into the input populations of each transformation according to Equ. (5). Note that the spatial transformation does not need the orientation of the demonstrator's body. The input populations also receive an external modulation applied asymmetrically to each stream. According to the task instructions, the inputs of the relevant network receive a positive modulation, whereas those of the other receive inhibition, i.e., $h^{\text {Task }}$ and $-\left(h^{\text {Task }}+\Delta h\right)$, respectively, where $h^{\text {Task }}>0$ is a constant, and $\Delta h \in\left\{0, \Delta h_{0}\right\}$. When $\Delta h=\Delta h_{0} \gg 0$, the network corresponding to the irrelevant transformation is completely inhibited. This case, where only the relevant transformation is active, is considered as the baseline condition.

The spatial transformation consists of mapping the orientation of the demonstrator's arm with the imitator's left and right arm, regardless of the demonstrator's body. For a given arm, two neural populations are required. The former receives the visual input and is connected to the latter through synaptic projections. Using Eqs. (1) and (5), the correct mapping functions for the left and the right arm are given by $\boldsymbol{M}=\boldsymbol{M}^{\mathrm{L}, \mathrm{Sp}}$ and $\boldsymbol{M}=\boldsymbol{M}^{\mathrm{R}, \mathrm{Sp}}$, respectively, where

$$
\boldsymbol{M}^{\mathrm{L}, \mathrm{Sp}}=\left\{\begin{array}{ll}
\operatorname{diag}(1,1,-1) & \boldsymbol{s}_{z}^{\mathrm{Arm}}<0 \\
\boldsymbol{I} & \text { otherwise }
\end{array} \quad \text { and } \quad \boldsymbol{M}^{\mathrm{R}, \mathrm{Sp}}=\operatorname{diag}(-1,1,1) \boldsymbol{M}^{\mathrm{L}, \mathrm{Sp}}\right.
$$


Since the frames of references of each arm are symmetric, so are the mapping matrices.

The anatomical transformation requires the combination of the orientation of the demonstrator's arm and that of his/her body. Neurophysiological data suggest that such a transformation is performed through gain fields, which are neural populations combining inputs from several external sources [13]. We define a gain field as a continuous set of neural fields denoted by GF $\phi$, where each of them is preferentially tuned to a specific body orientation $\phi$. The population encoding the demonstrator's arm orientation projects to each of them using Equ. (5) with mapping function $\boldsymbol{M}=\boldsymbol{R}_{y}(-\phi)$, where $\boldsymbol{R}_{y}(-\phi)$ is the rotation matrix around axis $\mathrm{Y}$ with angle $-\phi$. The body orientation is fed to the subfields through their modulatory input $h^{\mathrm{GF} \phi}(t)$ according to Equ. (6), with $\boldsymbol{M}=\boldsymbol{I}$ and $r^{\mathrm{GF} \phi}=(\sin \phi, 0,-\cos \phi)$. The gain field projects to the output population of the transfromation by synaptic projections with $\boldsymbol{M}=\boldsymbol{I}$.

The response selection is performed by a neural field receiving projections from the output population of both transformations. The competition arises naturally as an effect of the network recurrent connectivity, producing a winnertake-all type of operation $[7,12]$. As illustrated in Figure 3, according to the intrinsic distance metric given by the breadth $\sigma$ of the recurrent connections, close and overlapping inputs tend to average whereas distant ones compete. In our model, since the output strength of both streams are asymmetrically balanced, the correct response is always selected by the network. The network also receives a go signal by means of its modulatory input. Prior to the presentation of the target posture, $h^{\mathrm{Sel}}(t)=-h^{\mathrm{Go}} \ll 0$ so that the neural field is completely inactive. When the target posture is presented, the network is uninhibited, i.e., $h^{\mathrm{Sel}}(t)=0$, and the selection process begins. The network response is read-out using the population vector (Equ. (4)), which directly represents the selected arm posture in the frame of reference of the imitator.

\section{Results}

We simulated the two experiments described in Section 2.1. The demonstrator's arm and body postures, were systematically varied across each trial during both experiments ${ }^{1}$. Moreover, in experimental conditions involving the use of the left arm, the subnetwork corresponding to the right arm was not considered, and vice versa.

\footnotetext{
${ }^{1}$ The respective range of arm and body orientations are $\theta^{D} \in\left\{k \cdot 22.5^{\circ} \mid k \in\{0 . .8\}\right\}$ and $\phi^{D} \in\left\{k \cdot 22.5^{\circ} \mid k \in\{0 . .15\}\right\}$. The amplitude of postural change in Exp. 2 is in the range $\Delta \theta^{D} \in\left\{k \cdot 22.5^{\circ} \mid k \in\{1 . .8\}\right\}$. The model parameters are: the amplitudes of the weights, $\alpha^{R}=12, \alpha^{\mathrm{S}_{\mathrm{Arm}}, \mathrm{S}_{\mathrm{Out}}}=\alpha^{\mathrm{A}_{\mathrm{Arm}}, \mathrm{GF} \phi}=\alpha^{\mathrm{GF} \phi, \mathrm{A}_{\mathrm{Out}}}=5.4, \alpha^{\mathrm{A}_{\mathrm{Body}}, \mathrm{GF} \phi}=$ 8.0 , and $\alpha^{\mathrm{A}_{\mathrm{Out}}, \mathrm{Sel}}=\alpha^{\mathrm{S} \mathrm{Out}, \mathrm{Sel}}=5.0$; the breadth of the weights profiles and their offset, unless specified, $\sigma=0.5$ and $\eta=\oint_{\Gamma} g\left(\boldsymbol{r}, \boldsymbol{r}^{\prime}\right) \mathrm{d} \boldsymbol{r}$, then $\sigma^{\mathrm{A}_{\mathrm{Body}}, \mathrm{GF} \phi}=\infty$ and $\eta^{\mathrm{A}_{\mathrm{Arm}}, \mathrm{GF} \phi}=1.0$; the amplitude of the inputs, $\beta^{\mathrm{S}_{\mathrm{Arm}}}=\beta^{\mathrm{A}_{\mathrm{Arm}}}=\beta^{\mathrm{A}_{\mathrm{Body}}}=0.5$. These parameters were chosen so that the response energy of both transformations are equal for an equivalent task modulation. Finally, the task modulatory inputs and go signal are, $h^{\text {Task }}=0.5, \Delta h_{0}=0.75$ and $h^{\text {Go }}=1.5$.
} 


\subsection{Reaction Times and Accuracy}

The mean reaction times and the errors resulting from the transformations were measured in both experiments. Reaction times (RT) were defined as the time when the response energy $E(t)$ of the selection network reached a given threshold, whereas the transformation errors (Err) were defined as the angular distance between the population vector response $\hat{\boldsymbol{p}}(t)$ after network convergence, and the correct target position. Moreover, since we do not model the dynamics of arm movements, reaction times should be considered to be times of movement initiation rather than times of movement completion.
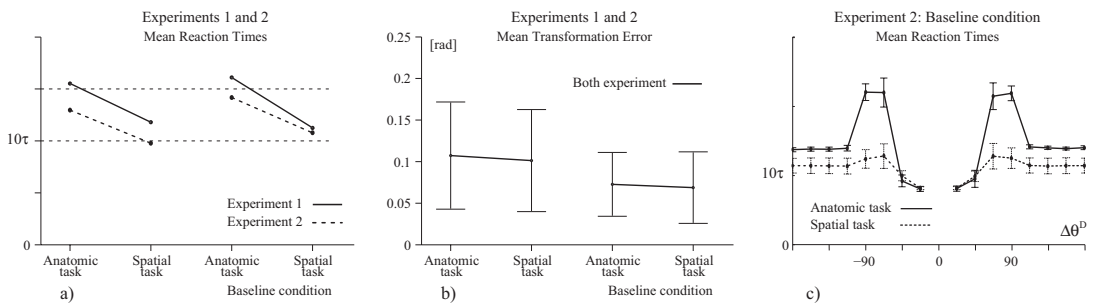

Fig. 5. a) Mean reaction times and b) transformation errors observed in both experiments. c) Reaction times during Experiment 2 in the baseline conditions shown according to the amount of postural change $\Delta \theta^{D}$.

As reported in Figure 5a, in both experiments, the average reaction times in anatomical conditions were longer than in the spatial task. Indeed, the former transformation requires more computations. In addition, a slight, but not significant increase in average reaction times can be noticed when comparing the normal condition with the baseline. Nevertheless, a difference between these conditions was observed on the transformation errors (see Fig. 5b). Indeed, a competition between the parallel transformations results in larger errors. In Experiment 2 , the amplitude of the postural change $\Delta \theta^{D}$ was different across trials. The reaction times dependency on this experimental variable in the baseline conditions is shown in Figure 5c. For small postural changes, reaction times were longer, but then decreased for larger $\Delta \theta^{D}$. This effect is caused by the center-surround recurrence in the neural dynamics, resulting in longer convergence times when moving from one attractor state to another, which is sufficiently close.

\subsection{Interference Patterns}

Then, we were interested in determining the interference patterns resulting from the competition between the two transformations. The reaction times and transformation errors were considered relative to the baseline conditions. Let us denote them, respectively, by $\Delta \mathrm{RT}=\mathrm{RT}-\mathrm{RT}_{0}$, and $\Delta \mathrm{Err}=\mathrm{Err}-\mathrm{Err}_{0}$, where $\mathrm{RT}_{0}$ and

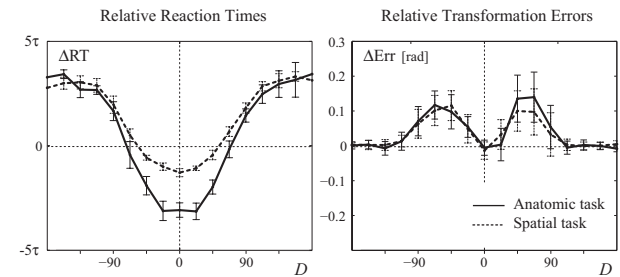

Fig. 6. Results of Experiment 1: Reaction times and transformation errors relative to the baseline condition are shown. 
Err $_{0}$ correspond to the reaction times and errors measured in the baseline conditions. In Figure 6, data from Experiment 1 are given according to the discrepancy $D$ between the responses of the anatomical on one hand, and the spatial transformation on the other hand. First, since the processing time of the spatial transformation is shorter, it interferes earlier with the anatomical transformation, and conversely. As an effect, the strength of the interferences on reaction times were globally higher in anatomical conditions. Next, the reaction times increased with the discrepancy between the responses, whereas transformation errors behaved slightly differently. The errors did also increase with the discrepancy, but only within a small range. For outermost distances, they decreased until approximately zero. This effect is the result of the averaging of close responses on the neural field. Similar effects were observed in Experiment 2 (see Fig. 7),
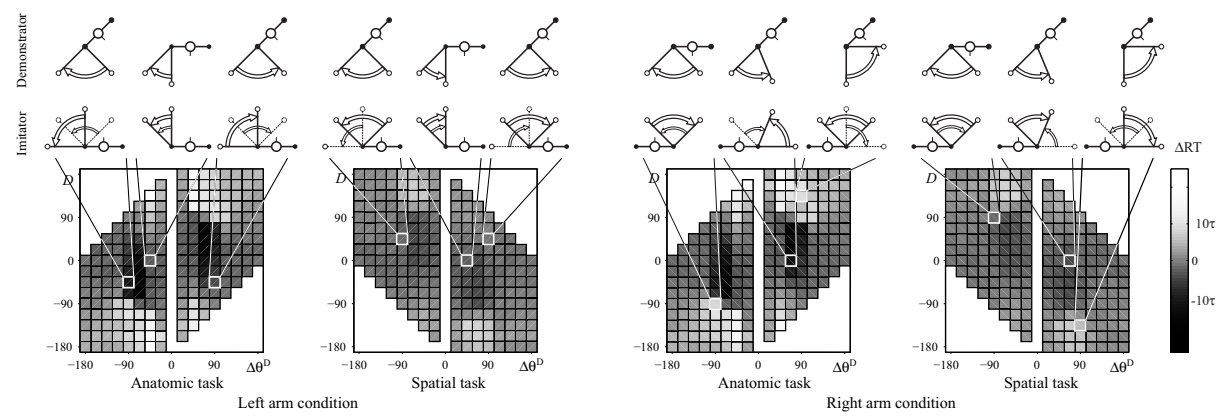

Fig. 7. Results of Experiment 2: Reaction times relative to the baseline condition. On top of each plot, examples of experimental conditions are shown. In each case, the largest arrow corresponds to the response of the relevant transformation.

i.e., the interference patterns were globally more important under anatomical conditions and the error patterns also depended on the discrepancy between the responses. Further, the interference patterns on reaction times exhibited a combination of the effects of both the discrepancy $D$ between the responses and the amount $\Delta \theta^{D}$ of arm postural change that were shown earlier in Figure $5 \mathrm{c}$ and 6. In conditions close to ideal congruency between the transformations, a general facilitatory effect was primarily produced which was even stronger for mid-range distances. In addition, an interaction between both variables on reaction times was observed. It produced a small shift of the interference pattern relative to the discrepancy $D$, which depended on $\Delta \theta^{D}$. In anatomical conditions, when the response of the spatial transformation is in the course of that of the anatomical transformation, the facilitory effect is strengthened, whereas when the former is located at a distance, it is weakened. Since this dependency between the responses is primarily caused by the difference in processing times, its effect is reversed in spatial conditions. Finally, because the errors were measured after network convergence, they were not different from those reported in Experiment 1 (see Fig. 6). 


\section{Discussion}

In this paper, we have presented a biologically-inspired neural model addressing the problem of transformations across frames of reference in a posture imitation task. Our modeling is based on the hypothesis that such an imitation process is mediated by two concurrent transformations, corresponding to the spatial and the anatomical imitative strategies $[4,5,12]$. We also devised an experimental paradigm which allowed us to measure the interference patterns that the interaction between the anatomical on one hand, and the spatial imitative strategy on the other hand produced. In addition, we also assumed that separate instances of the pair of transformations are responsible for the control of each side of the body. Since our experiments did not involve the use of both arms simultaneously, this latter hypothesis does not rule out the fact that the processes of each arm may be coupled and located within a single brain region [1,9]. As such, our results provide predictions of real behavioral responses.

Similar to other works which applied the Dynamic Field approach [7,12], our work goes beyond usual binary models, often proposed in experimental psychology [14]. Besides the fact that this framework allows the modeling of continuous stimulus variables and responses, which are more common in imitative behaviors, it is of high biological significance. Neurophysiological studies have shown that, in the superior temporal sulcus, body and arm postures are encoded into neural populations where each neuron exhibits tuning to a specific posture [2]. Similarly distributed representations, and correlates of decisional processes have also been reported in many other sensorimotor brain areas $[6,10,13]$. Together, these findings strengthen our approach by grounding it on a strong biological basis.

Behavioral studies on imitation report greater interferences during tasks where the spatial transformation is irrelevant, as compared to tasks where anatomical imitation has to be avoided [4,5,8]. Our model supports this observation, but explains it in terms of the longer processing time required by the anatomical imitative strategy, which needs to process an additional variable. Usual accounts for the greater influence of the spatial transformation consider primarily a stronger linkage with the decisional process [4,5,9,14]. Although both hypotheses are compatible, one may be interested in determining their respective influence, which would need more investigations.

Our modeling study also showed that combining of transformations produces interferences. One may wonder why the nervous system would use a combination of two strategies for solving imitation tasks since they produce interferences. Our simulations show that, in specific conditions, their interaction result in positive effects. For instance, when the imitator and the demonstrator are face to face, mirror imitation is faster, whereas anatomical imitation is more effective when the imitator looks at the back of the demonstrator. From this, we can propose an alternative hypothesis explaining that, in unconstrained conditions and when people are facing each other, mirror imitation is the most usual strategy for copying meaningless gestures [3]. Rather than assuming that mirror imitation has a stronger influence on the selection process $[4,8,9]$, we suggest that this strategy is the one which exhibits the maximal congruency between the con- 
current transformations. Additional neurophysiological evidence supporting our hypotheses can be found in an fMRI study showing that some of the brain areas activated during the imitation of finger movements are more active during specular than during anatomical imitation [9]. In this experiment, the authors did not consider the hypothesis that an anatomical mapping could exist between contralateral hands. The mirror condition which they showed to produce higher brain activation, corresponds in our approach to a condition where the responses of the parallel strategies are perfectly congruent. Since this case is effectively the one in which our model produces responses with the highest energy, the nervous system may hence be naturally biased toward this strategy.

Acknowledgements: This work was supported by the Swiss National Science Foundation, through grant no $620-066127$ of the SNF Professorships program.

\section{References}

1. M. Arbib, A. Billard, M. Iacoboni, and Oztop E. Mirror neurons, imitation and (synthetic) brain imaging. Neural Networks, 13:953-973, 2000.

2. E. Ashbridge, D. I. Perrett, M. W. Oram, and T. Jellema. Effect of image orientation and size on object recognition: responses of single units in the macaque monkey temporal cortex. Cognitive Neuropsychology, 17:13-34, 2000.

3. H. Bekkering, A. Wohlschlger, and M. Gattis. Imitation of gestures in children is goal-directed. Quarterly Journal of Experimental Psychology, 53:153-164, 2000.

4. B. I. Bertenthal, M. R. Longo, and A. Kosobud. Imitative response tendencies following observation of intransitive actions. Journal of Experimental Psychology: Human Perception and Performance, 32(2):210-225, 2006.

5. M. Brass, H. Bekkering, A. Wohlschläger, and W. Prinz. Compatibility between observed and executed finger movements: comparing symbolic, spatial and imitative cues. Brain and Cognition, 44:124-143, 2000.

6. P. Cisek and J. F. Kalaska. Neural correlates of reaching decision in dorsal premotor cortex: specification of multiple direction choices and final selection of action. Neuron, 45:801-814, 2005.

7. W. Erlhagen and G. Schöner. Dynamics field theory of movement preparation. Psychological Review, 109(3):545-572, 2002.

8. C. Heyes and E. Ray. Spatial S-R compatibility effects in an intentional imitation task. Psychonomic Bulletin \& Review, 11(4):703-708, 2004.

9. L. Koski, M. Iacoboni, M.-C. Dubeau, R. P. Woods, and J. C. Mazziotta. Modulation of cortical activity during different imitative behaviors. Journal of Neuophysiology, 89:460-471, 2003.

10. T. N. Aflalo and M. S. A. Graziano. Partial tuning of motor cortex neurons to final posture in a free-moving paradigm. Proceedings of the National Academy of Sciences USA, 103(8):2909-2914, 2006.

11. G. Rizzolatti, L. Fogassi, and V. Gallese. Neurophysiological mechanisms underlying the understanding of actions. Nature Reviews Neuroscience, 2:661-670, 2001.

12. E. L. Sauser and A. G. Billard. Parallel and distributed neural models of the ideomotor principle: An investigation of imitative cortical pathways. Neural Networks, 19(3):285-298, 2006.

13. H. Scherberger and R. A. Andresen. Sensorimotor transformations. The Visual Neurosciences. MIT Press, pages 1324-1336, 2003.

14. H. H. Zhang, J. Zhang, and S. Kornblum. A parallel distributed processing model of stimulus-stimulus and stimulus-response compatibility. Cognitive Psychology, 38:386-432, 1999. 